\title{
Knockdown of RAC1 and VASP gene expression inhibits breast cancer cell migration
}

\author{
YIHAO TIAN $^{1,2}$, LIU XU ${ }^{1}$, YANQI HE ${ }^{1}$, XIAOLONG XU ${ }^{1}, \mathrm{KAI} \mathrm{LI}^{1}$, \\ YANBIN MA ${ }^{1}$, YANG GAO ${ }^{1}$, DEFEI WEI $^{1}$ and LEI WEI ${ }^{1}$ \\ ${ }^{1}$ Department of Pathology and Pathophysiology, Hubei Provincial Key Laboratory of Developmentally Originated Disease; \\ ${ }^{2}$ Department of Human Anatomy and Histology and Embryology, School of Basic Medical Sciences, \\ Wuhan University, Wuhan, Hubei 430071, P.R. China
}

Received November 11, 2017; Accepted April 13, 2018

DOI: $10.3892 / \mathrm{ol} .2018 .8930$

\begin{abstract}
The ability of tumor cells to migrate is biologically fundamental for tumorigenesis, growth, metastasis and invasion. The present study examined the role of Ras-related C3 botulinum toxin substrate (RAC1) and vasodilator-stimulated phosphoprotein (VASP) in breast cancer cell migration. According to data in Kaplan, Oncomine and The Cancer Genome Atlas, increased expression levels of RAC1 and VASP in breast cancer are associated with decreased cancer cell differentiation, advanced pathological stage and more aggressive tumor subtypes, while increased VASP mRNA expression levels are positively correlated with a poor prognosis in patients with breast cancer. The short hairpin (sh)RNA technique was employed to knock down the expression of RAC1 or VASP. Stable interference with the expression of RAC1 or VASP using RAC1-shRNA or VASP-shRNA, respectively, was established in MCF-7 breast cancer cells. In RAC1-shRNA or VASP-shRNA cells, the protein expression levels of RAC1 or VASP were significantly downregulated compared with control cells. The proliferation and migration rates of the RAC1-shRNA or VASP-shRNA cells were significantly lower compared with control cells. It was observed that the protein expression levels of VASP also decreased in RAC1-shRNA cells compared with control cells. The results revealed that RAC1 and VASP may serve important roles in promoting the migration of MCF-7 breast cancer cells, and that VASP may among the downstream signaling molecules associated with RAC1.
\end{abstract}

Correspondence to: Professor Lei Wei, Department of Pathology and Pathophysiology, Hubei Provincial Key Laboratory of Developmentally Originated Disease, School of Basic Medical Sciences, Wuhan University, 185 Donghu Road, Wuhan, Hubei 430071, P.R. China

E-mail: leiwei@whu.edu.cn

Key words: MCF-7, Ras-related C3 botulinum toxin substrate 1, vasodilator-stimulated phosphoprotein, breast cancer cell, migration, short hairpin RNA

\section{Introduction}

According to Chinese cancer statistics in 2015 (1), thyroid cancer is the most commonly diagnosed cancer type in women aged $<30$ years, followed by breast cancer at age 30-59 years. Invasion and metastasis are the leading causes of mortality in patients with breast cancer (2). Therefore, in addition to surgery, chemotherapy, radiotherapy and targeted therapy treatments, finding effective ways to improve the treatment of patients with cancer is of primary concern.

Vasodilator-stimulated phosphoprotein (VASP), a member of the Enabled (Ena)/VASP family, is an actin-associated protein. VASP is mainly distributed at locations associated with adhesion and migration, including the anterior border of the lamellar parapodium, filopodia cacumen and adhesion plaque, and has a highly dynamic position in the cellular membrane (3). It is involved in regulating actin filament rearrangement in the process of cell adhesion and expansion, and is associated with cell adhesion, cytomorphosis and mobility (4,5). According to Krause et al (3), an increase in VASP expression promotes the movement of the Listeria bacteria in vitro. VASP induces the assembling of actin via binding of ActA, which accelerates the movement of the Listeria bacterium inside cells. Furthermore, Sechi et al (6) reported that deleting the domain of ActA that directly combines with VASP, effectively suppresses the movement of the Listeria bacterium inside cells. In eukaryotic cells, the expression levels of VASP are positively correlated with the formation speed of lamellipodia in mouse melanoma B16-F1 cells (6). Notably, overexpression of VASP in wild-type NIH 3T3 cells resulted in malignant transformation of the cells (7). It has also been revealed that increased expression levels of VASP are correlated with decreased cellular differentiation and an increased pathological stage of lung tumors (8). These data suggested that VASP may serve a crucial role in regulating cellular migration, in addition to tumorigenesis and development.

Rho GTPases [Rho, Ras-related C3 botulinum toxin substrate (Rac)1 and cell division control protein 42 homolog (Cdc42)] are important signaling molecules that are involved in regulating cell migration through cellular cytoskeletal rearrangement $(9,10)$. The activation of $\mathrm{RAC1}$, one member of the 
family, promotes the recruitment of the integrin family, participates in the assembling of the cytoskeleton, and induces the accumulation and polarization of tumor invasion (11). However, as a key factor in the regulation of cell migration, the relative contribution of RAC1 to cytoskeletal rearrangement depends on specific conditions and cell type. It was revealed that there were no significant changes in cellular migratory abilities in RAC1-deficient rat fibroblasts and macrophages (12). However, downregulation of RAC1 was demonstrated to result in the inhibition of migration in various types of cells, including gastric, testicular and colorectal carcinoma cells (13). Notably, regarding the association between RAC1 and VASP, Fryer et al (14) reported that in human umbilical vein endothelial cells and HeLa cells, p21-activated kinase (PAK; serine 21), a downstream molecule of RAC1 phosphorylated by cGMP-dependent protein kinase (PKG), promoted the formation of Pak/VASP and thereby affected cell motility.

Therefore, the present study focused on examining the role of RAC1 and VASP in the development of breast cancer, by analyzing data in Kaplan, Oncomine and The Cancer Genome Atlas (TCGA), and using in vitro experiments in breast cancer MCF-7 cells.

\section{Materials and methods}

Online databases. Patients with breast cancer were divided into a low expression level group and a high expression level group, according to the median RAC1 or VASP mRNA expression levels (15). Data from the Kaplan (http://kmplot. com/analysis/index.php? $\mathrm{p}=$ service \&cancer=breast) (Data set no. Affy ID, 202205_at VASP; Affy ID, 208640_at p21-RAC1, RAC1 and TC-2), Oncomine (https://www.oncomine. com/resource/login.html) and TCGA (http://xena.ucsc. edu/public-hubs/) databases were used for correlation analysis of different gene expression levels and patient clinical information.

Analysis of the association between VASP, RAC1 and molecular subtypes of breast cancer based on data from the TCGA database. The clinical information data of patients with breast cancer were collected from TCGA and analyzed using UCSC Xena (http://xena.ucsc.edu/\#analyze; University of California, Santa Cruz, CA, USA). The mRNA expression level of RAC1 and VASP in normal breast cancer tissue was the lowest, increasing gradually in the Luminal A, Luminal B, BLBC and Her-2 enrichment subtype groups.

Cell culture. The breast carcinoma MCF-7 cell line and the 293T cell line were purchased from the Department of Pathophysiology, Medical College of Wuhan University (Wuhan, China). Cells were cultured in Dulbecco's modified Eagle's medium (DMEM; Hyclone; GE Healthcare, Logan, UT, USA) supplemented with $10 \%$ newborn calf serum (Gibco; Thermo Fisher Scientific, Inc., Waltham, MA, USA) and $1 \%$ antibiotics $(100 \mathrm{U} / \mathrm{ml}$ penicillin $\mathrm{G}$ and $100 \mathrm{U} / \mathrm{ml}$ streptomycin sulfate) at $37^{\circ} \mathrm{C}$ in an environment containing $5 \% \mathrm{CO}_{2}$. Cells were passaged at 70-80\% confluence using $0.02 \%$ EDTA in PBS and $0.25 \%$ trypsin.

Construction of short hairpin (shRNA) MCF-7 stable cell lines. RAC1 and VASP stable knockdown in the MCF-7 cell line was established via lentiviral-based stable shRNA overexpression. The target sequences for RAC1, 5'-GATCCGGAA GGAGATTGGTGCTGTAAAACTCGAGAAAATGTCGTG GTTAGAGGAATTTTTG-3'; VASP, 5'-CCGGAGGAATTG CAGAAAGTGAAAGCTCGAGCTTTCACTTTCTGCAAT TCCTTTTTTG-3'; and shRNA control, 5'-CAACAAGAT GAAGAGCACCAA-3' were designed by Sigma-Aldrich; Merck KGaA (Darmstadt, Germany) and constructed into lentiviral-based green fluorescent protein-shRNA expression plasmids, pLVshRNA-EGFP-Puro (Beijing Inovogen Tech Co., Ltd., Beijing, China). The lentiviruses ( $4 \mu \mathrm{g})$ were packaged into the $293 \mathrm{~T}$ cell line. The cell culture supernatant was collected 48 and $72 \mathrm{~h}$ after transfection, centrifuged at $3,000 \mathrm{x}$ g for $15 \mathrm{~min}$, filtered through a $0.45-\mu \mathrm{m}$ polyvinylidene difluoride filter (Acrodisc) to remove cellular debris, aliquoted into Eppendorf tubes and stored at $-70^{\circ} \mathrm{C}$ as the viral solution for use. MCF-7 cells were plated in a 96-well plate at a density of $1 \times 10^{4}$ cells/well. After $24 \mathrm{~h}$, the viral solution was added to the MCF-7 cells and $10 \mu \mathrm{g} / \mathrm{ml}$ Polybrene (Sigma Aldrich; Merck KGaA) was added to increase the efficiency of infection. The medium containing the virus was removed $12 \mathrm{~h}$ later and replaced with $200 \mu \mathrm{l}$ DMEM supplemented with $10 \%$ fetal bovine serum. The cell lines were screened using cell medium with $2 \mu \mathrm{g} / \mathrm{ml}$ puromycin. The infection efficiency of the virus was further confirmed by fluorescence microscopy (magnification, x200). The interference efficiency of the cells was detected by reverse transcription-polymerase chain reaction (RT-PCR) and western blotting.

$R T-P C R$. To determine the mRNA expression level of RAC1 and VASP in MCF-7 cells, total RNA was purified using a total RNA isolation system (Promega Corporation, Madison, WI, USA) and RT-PCR was performed using the Access RT-PCR system, according to the manufacturer's protocol (Promega Corporation). The primers used were as follows: RAC1 forward, 5'ATGTCCGTGCAAAGTGGTATC3' and reverse, 5'CTCGGATCGCTTCGTCAAACA3'; VASP forward, 5'-CTGGGAGAAGAACAGCACAACC-3' and reverse, 5'-AGGTCCGAGTAATCACTGGAGC-3'; GAPDH forward, 5'-TGATGACATCAAGAAGGTGGTGAAG-3' and reverse, 5'-TCCTTGGAGGCCATGTGGGCCAT-3'. The thermocycling conditions were as follows: $\mathrm{RAC1}, 94^{\circ} \mathrm{C}$ for $40 \mathrm{sec}$; $54^{\circ} \mathrm{C}$ for $30 \mathrm{sec}$ and $72^{\circ} \mathrm{C}$ for $30 \mathrm{sec}$ for 30 cycles; VASP, $94^{\circ} \mathrm{C}$ for $40 \mathrm{sec} ; 60^{\circ} \mathrm{C}$ for $30 \mathrm{sec}$ and $72^{\circ} \mathrm{C}$ for $30 \mathrm{sec}$ for 30 cycles. GAPDH was employed as an internal control for mRNA and protein analyses.

Western blot analysis. The MCF-7 cells were washed twice with PBS and lysed in ice-cold radioimmunoprecipitation assay buffer (1.5 mM MgCl $2,1 \mathrm{mM}$ EGTA, $1 \mathrm{mM}$ PMSF, $10 \mathrm{mM} \mathrm{NaVO}_{3}$ and $10 \mathrm{mM}$ PIC), using $100 \mu \mathrm{l}$ lysis buffer per well. Following $10 \mathrm{~min}$ on ice, the lysates were transferred into microcentrifuge tubes and centrifuged at $12,000 \mathrm{x} \mathrm{g}$ for $15 \mathrm{~min}$ at $4^{\circ} \mathrm{C}$. Following $12 \%$ SDS-PAGE separation, proteins were electrotransferred to a nitrocellulose membrane for $90 \mathrm{~min}$ for RAC1 and GAPDH, and $120 \mathrm{~min}$ for VASP. Subsequent to blocking with 5\% BSA in Tris-buffered saline (TBS) at $37^{\circ} \mathrm{C}$ for $1 \mathrm{~h}$, the bands were excised according to the protein marker (PageRuler Prestained Protein Ladder; cat. no. 00575411; Thermo Fisher Scientific, Inc.) protocol, 
and membrane strips were incubated with the following primary antibodies: Anti-RAC1 (cat. no. 610651; dilution, 1:1,000; BD Biosciences, Franklin Lakes, NJ, USA), anti-VASP (cat. no. 0010-10; dilution, 1:1,000; ImmunoGlobe GmbH, Himmelstadt, Germany) and anti-GAPDH (cat. no. SC-69778; dilution, 1:5,000; Santa Cruz Biotechnology, Inc., Dallas, TX, USA) overnight at $4^{\circ} \mathrm{C}$. Subsequent to 5 washes in TBS with Tween-20 (TBST) for $10 \mathrm{~min}$, the membrane strips were incubated with dilutions $(1: 10,000)$ of horseradish peroxidase-conjugated goat anti-mouse IgG (cat. no. 16473-1-AP; ProteinTech Group, Inc., Chicago, IL, USA) and anti-mouse IgG (cat. no. 10285-1-AP, ProteinTech Group, Inc.) at $37^{\circ} \mathrm{C}$ for $2 \mathrm{~h}$, and washed with 5 times in TBST for $10 \mathrm{~min}$. Following a further washing step, an enhanced chemiluminescence immune enzymatic reaction (Beijing Zoman Biotechnology Co., Ltd., Beijing, China) was performed to view the bands.

Scratch wound healing motility assays (2D-cell migration assays). Cells were cultured as mentioned earlier. Cells were seeded on 6-well plates and allowed to grow to $100 \%$ confluency. Confluent monolayers were scratched with a pipette tip and maintained in serum-free DMEM medium for $12 \mathrm{~h}$ and maintained in an incubator at $37^{\circ} \mathrm{C}$. The plates were washed once with fresh medium to remove non-adherent cells and images were captured using an inverted phase-contrast microscope (magnification, x200; Olympus Corporation, Tokyo, Japan). The cell migration rate was evaluated by calculating the average cell migration distance in $48 \mathrm{~h}$.

Transwell assays (3D-cell migration assays). A migration assay was performed using the Transwell system, which allows cells to migrate through a polycarbonate membrane with $8-\mu \mathrm{m}$ pores. Cells were trypsinized, washed and resuspended in serum-free DMEM at $1 \times 10^{6}$ cells $/ \mathrm{ml}$. This suspension $(100 \mu \mathrm{l})$ was added to the upper chamber of the Transwell. After an 8 -h incubation at $37^{\circ} \mathrm{C}$ in the presence of $5 \% \mathrm{CO}_{2}$, the lower chamber was filled with $500 \mu 1$ DMEM supplemented with $20 \%$ calf serum. Following incubation for $18 \mathrm{~h}$ at $37^{\circ} \mathrm{C}$ in the presence of $5 \% \mathrm{CO}_{2}$, the cells were fixed for $30 \mathrm{~min}$ in $4 \%$ formaldehyde and stained at room temperature for $15 \mathrm{~min}$ with $5 \%$ crystal violet. The filters were then rinsed thoroughly in distilled water and checked by bright-field microscopy (magnification, $\mathrm{x} 200$ ) to ensure that the cells were adherent and had migrated. The non-migrating cells were then carefully removed from the upper surface (inside) of the Transwell with a wet cotton swab. To quantify cell motility, cells that had migrated to the bottom surface of the filter were counted. A total of nine evenly spaced fields of cells were counted in each well, using an inverted phase-contrast microscope at magnification, $\mathrm{x} 200$.

Cell Counting kit-8 (CCK-8) assay to detect apoptosis. Cells in the logarithmic growth phase were seeded onto 96 -well cell culture plates at a density of $\sim 4 \times 10^{3}$ cells $/ 1$, and $100 \mu$ l complete DMEM was added to each well. A total of 6 parallel wells were set up and the cells were incubated in a $37^{\circ} \mathrm{C}$-incubator for $24 \mathrm{~h}$. A total of $10 \mu \mathrm{l} \mathrm{CCK-8} \mathrm{solution} \mathrm{(Beijing} \mathrm{Zoman}$ Biotechnology Co., Ltd.) diluted in $90 \mu \mathrm{l}$ medium was added to each well. The cells were incubated at $37^{\circ} \mathrm{C}$ for $2 \mathrm{~h}$, and the absorbance nm was measured at a wavelength of 450 using a microplate reader.

Flow cytometry to detect apoptosis. The RAC1-shRNA and negative control (NC)-shRNA MCF-7 cells were cultured in a 6-well plate until the cells reached $60-70 \%$ confluency. A total of $1-5 \times 10^{5}$ cells were digested with EDTA-free trypsin and $500 \mu \mathrm{l}$ binding buffer (Beyotime Institute of Biotechnology, Haimen, China) to resuspend the cells. A total of $5 \mu 1$ Annexin V-fluorescein isothiocyanate and $10 \mu \mathrm{l}$ propidium iodide (both Beyotime Institute of Biotechnology) was added to each tube, and these were incubated at room temperature for 5 min prior to flow cytometry.

Statistical analysis. Data are expressed as the mean \pm standard error of the mean. Differences between groups were analyzed using one-way analysis of variance followed by a Bonferroni's post-hoc test. Kaplan-Meier analysis, followed by a Log rank test was performed to determine survival rates (http://kmplot.com/analysis/index. php? $\mathrm{p}=$ service\&cancer). The data of RAC1 and VASP mRNA expression in breast cancer tissues, and the clinical information of patients with breast cancer were collected from TCGA and analyzed using the UCSC Xena database (http://xena.ucsc. edu/\#analyze; University of California, Santa Cruz, CA, USA). The mRNA expression levels of RAC1 and VASP were quantified. $\mathrm{P}<0.05$ was considered to indicate a statistically significant difference.

\section{Results}

Expression of RACl and VASP mRNA is increased in breast cancer tissue data obtained from online databases. According to the data from Oncomine, the expression of RAC1 and VASP at the mRNA level in invasive ductal carcinoma tissues (1,556 cases) was significantly increased compared with that in control tissues (144 cases; $\mathrm{P}<0.05$, Fig. $1 \mathrm{~A}$ and $\mathrm{B}$ ). Furthermore, the patients with breast cancer were divided into a low expression level group and a high expression level group, according to the median RAC1 or VASP mRNA expression levels (15). The mortality rate of patients with breast cancer at 50 months in the low RAC1 expression group (883 cases) was higher than that in the high expression group (881 cases; $40.2 \%$ vs. $49 \%$; log rank, P>0.05; Fig. 1C). Correspondingly, the mortality rate of patients with breast cancer at 50 months in the in the high VASP expression group (1,971 cases) was significantly increased compared with the low expression level group (1,980 cases) (34.7\% vs. 37.8\%, log rank $\mathrm{P}<0.05$; Fig. 1D). This indicated that increased VASP mRNA expression levels were positively associated with poor prognosis in patients with breast cancer.

Association between the mRNA expression level of RACl or VASP in breast cancer tissues, and the subtype and staging of breast cancer. The mRNA expression levels of RAC1 and VASP in breast cancer tissues, and the clinical information of patients with breast cancer, were collected from TCGA and analyzed using UCSC Xena (http://xena.ucsc.edu/\#analyze; University of California, Santa Cruz, CA, USA). The mRNA expression levels of RAC1 and VASP were quantified. 

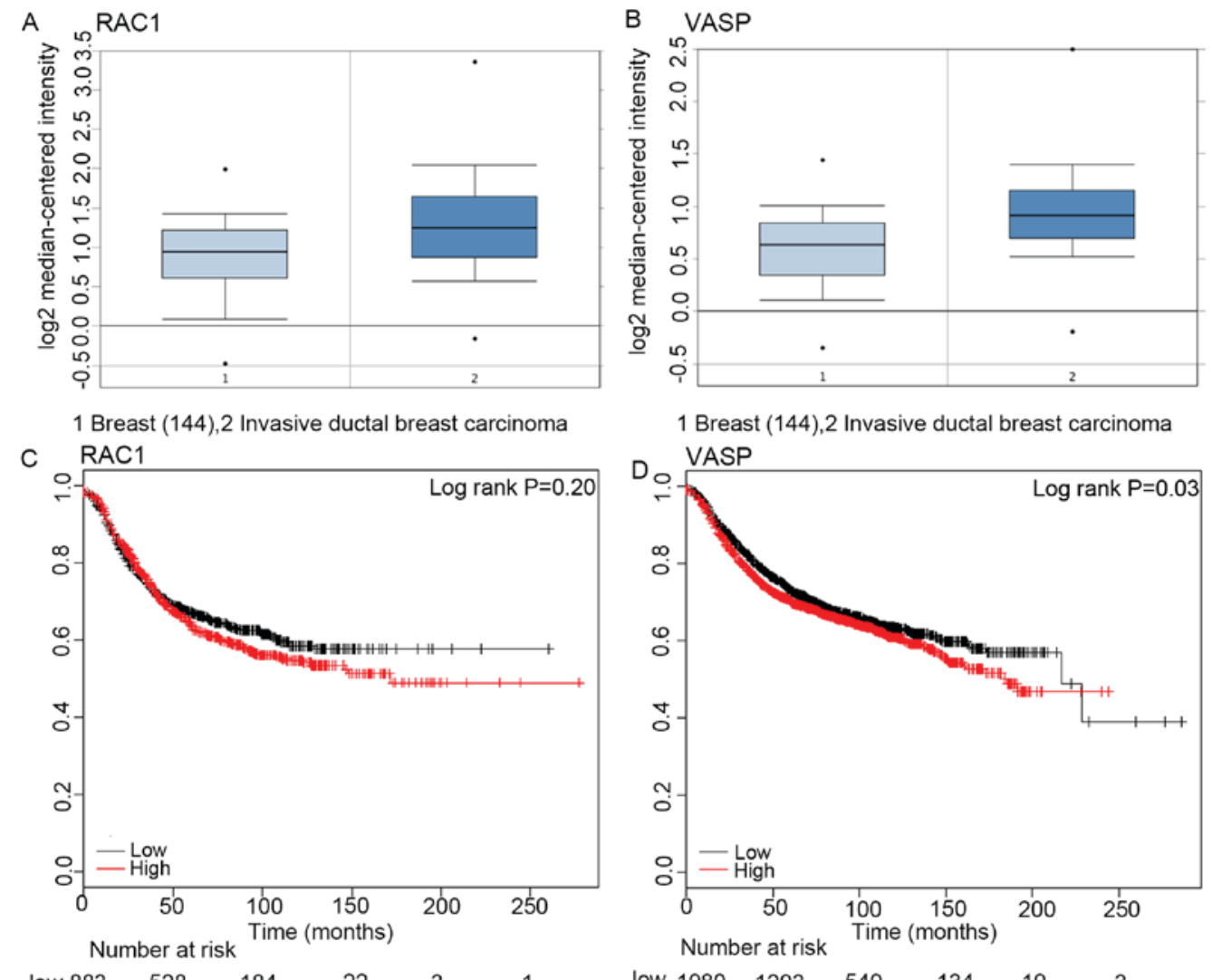

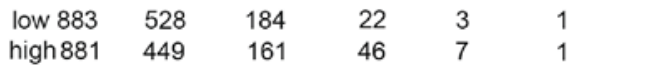

$\begin{array}{llllcl}\text { low } 1980 & 1293 & 549 & 134 & 19 & 3 \\ \text { high } 1971 & 1226 & 526 & 107 & 8 & 0\end{array}$

Figure 1. RAC1 and VASP mRNA expression in tumor and normal tissue. (A) RAC1 mRNA expression in human invasive ductal carcinoma and normal breast tissue. * $\mathrm{P}<0.05$. (B) VASP mRNA expression in human invasive ductal carcinoma tissue and normal breast tissue. * $\mathrm{P}<0.05$. (C) Expression of $\mathrm{RAC} 1$ mRNA in breast cancer tissue, and its association with the overall survival time of patients with breast cancer. P $>0.05$. (D) VASP expression in breast cancer tissue, and its association with the overall survival time of patients with breast cancer. ${ }^{*} \mathrm{P}<0.05$. RAC1, Ras-related C3 botulinum toxin substrate 1 ; VASP, vasodilator-stimulated phosphoprotein.

The Prosigna breast Cancer Prognstic Gene Signature Assay measures the expression level of 50 genes in surgically resected breast cancer samples to classify a tumor as one of four intrinsic subtypes (Luminal A, Luminal B, HER2-enriched, and Basal-like) (16). According to the Prosigna breast Cancer Prognstic Gene Signature Assay classification criteria, breast cancer is divided into Luminal A, Luminal B, Her-2 enrichment and Basal-like breast cancer (BLBC) subtypes. The control was the Normal group, in which the RAC1 mRNA expression level was the lowest, increasing gradually in the Luminal A, Luminal B, BLBC and Her-2 enrichment subtype groups. Furthermore, the expression in the Luminal A subtype group was significantly decreased compared with the BLBC subtype and Her-2 subtype groups $(\mathrm{P}<0.05$; Fig. 2A). The expression in the Luminal B subtype group was significantly decreased compared with the Her-2 enrichment subtype group (Fig. 2A; $\mathrm{P}<0.05)$.

According to the American Joint Committee on Cancer (AJCC) classification criteria $(17,18)$, the pathological staging of breast cancer was divided into 4 stages, and the mRNA expression level of $\mathrm{RAC} 1$ gradually increased from stage I-IV. The mRNA expression level of RAC1 in stage I was decreased compared with stages III and IV, with a significant difference $(\mathrm{P}<0.05$; Fig. 2B). However, elevated RAC1 mRNA expression was not associated with lymph node metastasis ( $\mathrm{P}>0.05$; Fig. 2C).
The mRNA expression level of VASP in the Normal group was the lowest, and its expression level gradually increased in the Luminal B, Luminal A, BLBC and Her-2 subtype groups. The mRNA expression level of VASP in the Luminal A subtype was significantly decreased compared with the Her-2-enriched and BLBC subtypes. However, it was increased compared with the Luminal B group ( $\mathrm{P}<0.05$; Fig. $3 \mathrm{~A})$. The mRNA expression level of VASP in the Luminal B subtype was significantly decreased compared with the Her-2 enriched subtype $(\mathrm{P}<0.05$; Fig. 3A). The expression of VASP mRNA gradually increased from stage I-IV ( $>>0.05$; Fig. 3B), however, elevated VASP mRNA expression was not associated with AJCC staging or lymph node metastasis ( $\mathrm{P}>0.05$; Fig. 3C).

VASP-shRNA interference in MCF-7 cells. The shRNA against the human VASP gene, or NC-shRNA were stably transfected into MCF-7 cells. Fluorescence microscopy revealed green fluorescence in the cytoplasm and nuclei, indicating successful transfection (Fig. 4). The expression level of VASP was detected on the mRNA and protein levels by RT-qPCR and western blotting, as presented in (Fig. 5A-C). Compared with the stable NC-shRNA MCF-7 cells, the inhibition rates of mRNA and protein expression levels of VASP in the stable VASP-shRNA MCF-7 cells were 78 and 65\%, respectively $(\mathrm{P}<0.05)$. These data indicated the establishment of stable VASP-shRNA MCF-7 cells. 

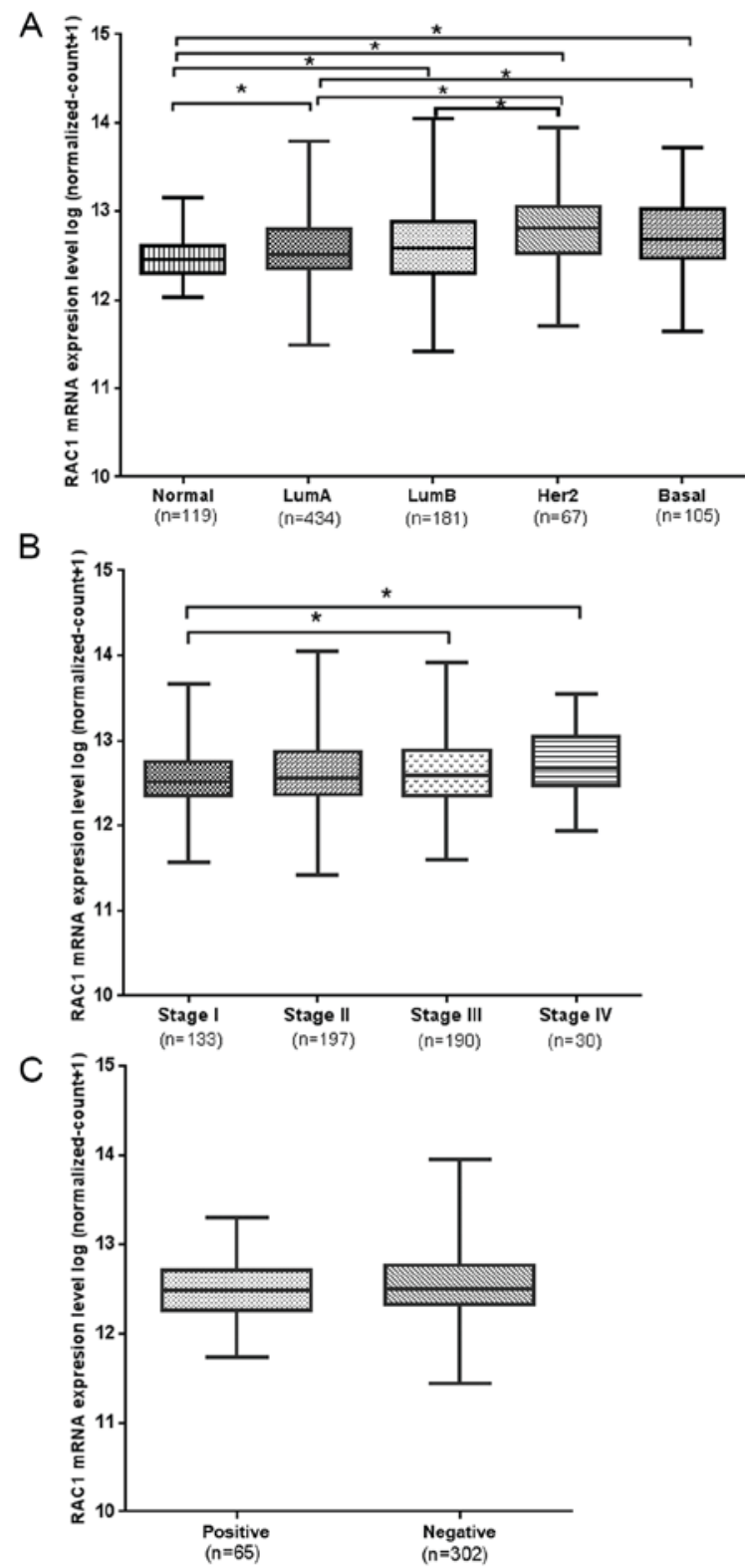

Figure 2. RAC1 mRNA expression in tumor tissue, and its association with the classification, staging and lymph node metastasis of breast cancer. (A) RAC1 mRNA expression in LumA, LumB, Her2, Basal and Normal tissue. ${ }^{*} \mathrm{P}<0.05$. (B) American Joint Committee on Cancer staging and RAC1 mRNA expression. ${ }^{*} \mathrm{P}<0.05$. (C) RAC1 mRNA expression in lymph node metastasis-positive and -negative breast cancer tissues. RAC1, Ras-related C3 botulinum toxin substrate 1; LumA, luminal A; LumB, luminal B; Her2, Her-2 enrichment; Basal, basal-like breast cancer.

RAC1-shRNA interference in MCF-7 cells. The shRNA against RAC1 was stably transfected into MCF-7 cells. Fluorescence microscopy revealed the presence of green fluorescence in the cytoplasm and nuclei (Fig. 4). The expression of RAC1 and VASP in the cells was detected on the mRNA and protein levels by RT-qPCR and western blotting (Fig. 5D-F). Compared with the stable NC-shRNA MCF-7 cells, the of the RAC1 and VASP mRNA expression-inhibition rate in the stable RAC1-shRNA MCF-7 cells was $86 \%(\mathrm{P}<0.05)$ and $67 \%$ $(\mathrm{P}<0.05)$, respectively. The RAC1 and VASP protein expression-inhibition rate of the stable RAC1-shRNA MCF-7 cells was $67 \%(\mathrm{P}<0.05)$ and $44 \%(\mathrm{P}<0.05)$, respectively. These data indicated the establishment of stable RAC1-shRNA MCF-7
A

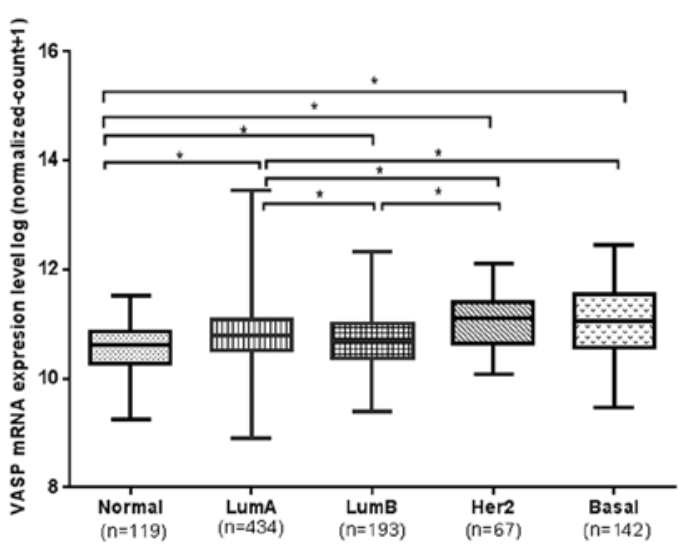

B

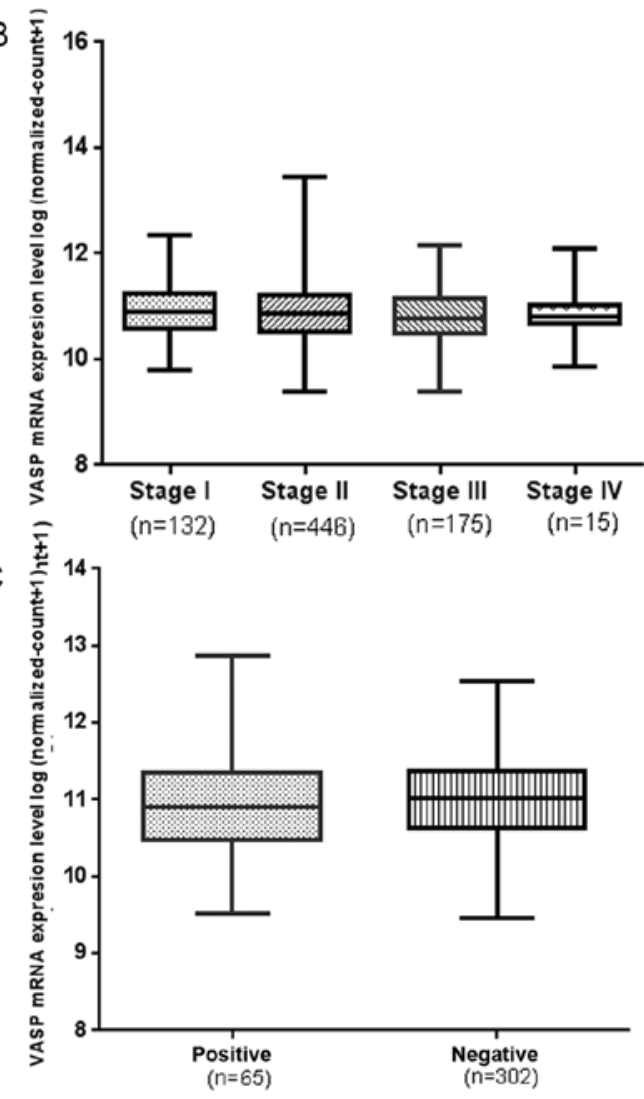

Figure 3. VASP mRNA expression in tumor tissue, and its association with the classification, staging and lymph node metastasis of breast cancer. (A) VASP mRNA expression in LumA, LumB, Her2, Basal and Normal tissue. ${ }^{*} \mathrm{P}<0.05$. (B) American Joint Committee on Cancer staging and VASP mRNA expression. $\mathrm{P}>0.05$. (C) VASP mRNA expression in lymph node metastasis-positive and -negative breast cancer tissues. VASP, vasodilator-stimulated phosphoprotein; LumA, luminal A; LumB, luminal B; Her2, Her-2 enrichment; Basal, basal-like breast cancer.

cells, and that RAC1-shRNA transfection interfered with the expression levels of VASP.

Effect of RACl and VASP-shRNA interference on MCF-7 cell proliferation. In order to study the effect of downregulation of RAC1 and VASP expression on the proliferation of MCF-7 cells, stable RAC1-shRNA, VASP-shRNA and NC-shRNA MCF-7 cells were cultured for $48 \mathrm{~h}$, and the proliferation rate of the cells was analyzed using a CCK-8 assay. The results revealed that the proliferation rate of 

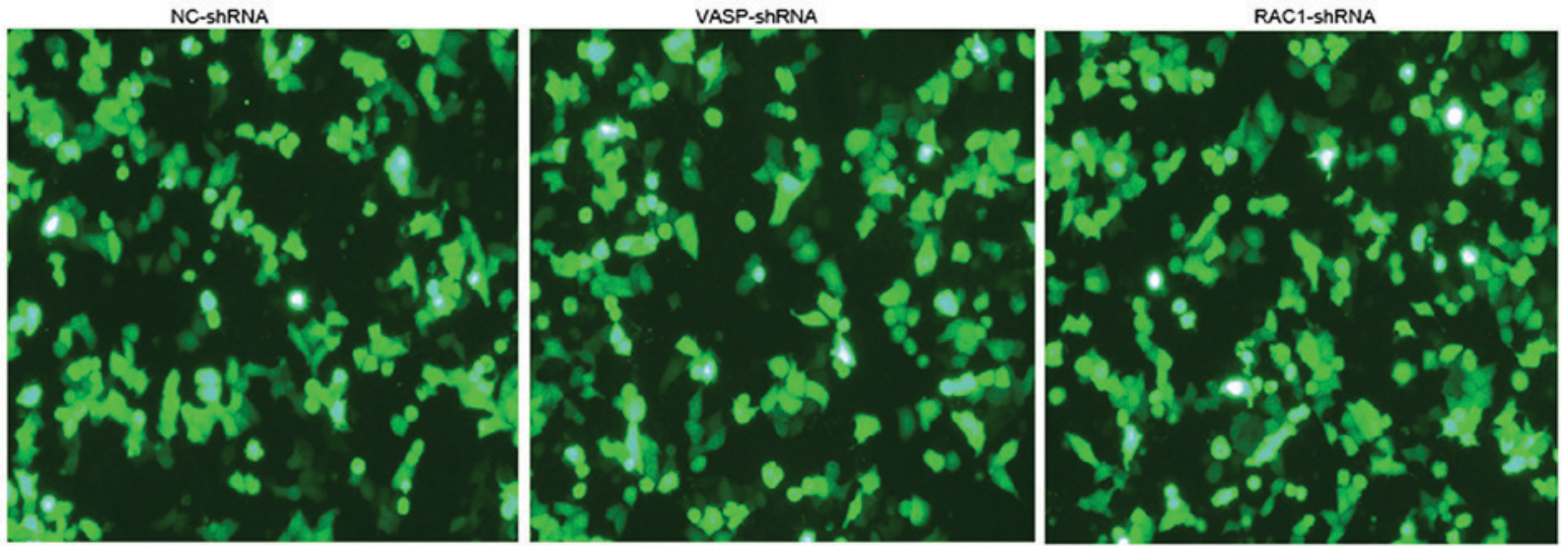

Figure 4. Expression of green fluorescent protein in VASP-shRNA, RAC1-shRNA and NC-shRNA MCF-7 cells, using an inverted phase-contrast microscope (magnification, x200; Olympus Corporation, Tokyo, Japan). VASP, vasodilator-stimulated phosphoprotein; RAC1, Ras-related C3 botulinum toxin substrate; shRNA, short hairpin RNA; NC, negative control.

A

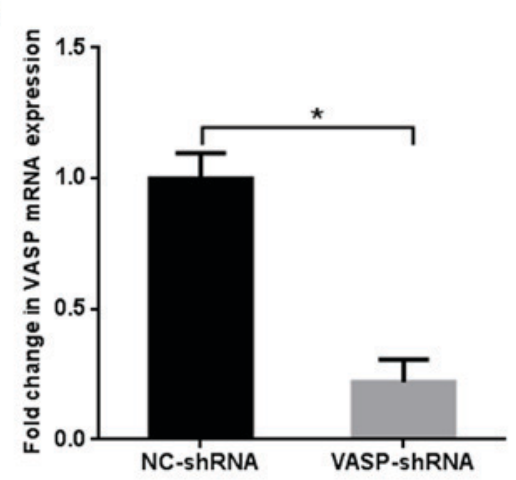

C

VASP

GAPDH

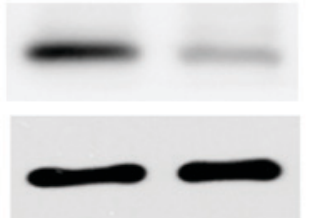

E

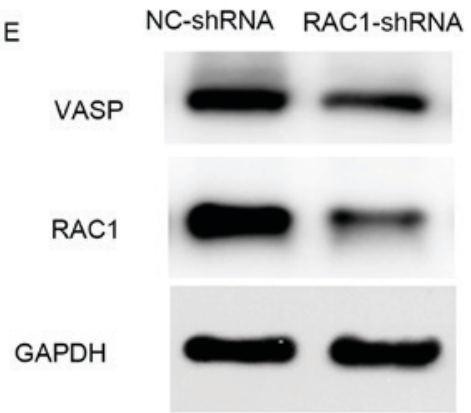

NC-ShRNA VASP-shRNA
B

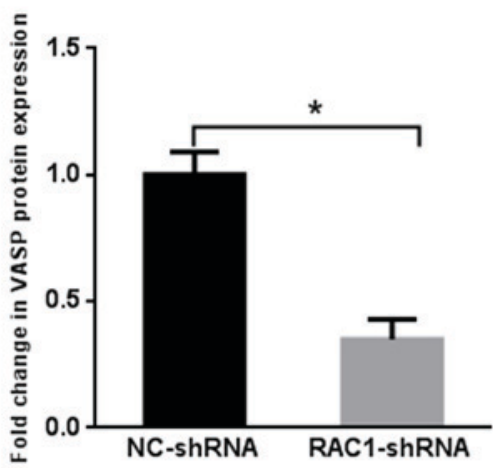

D
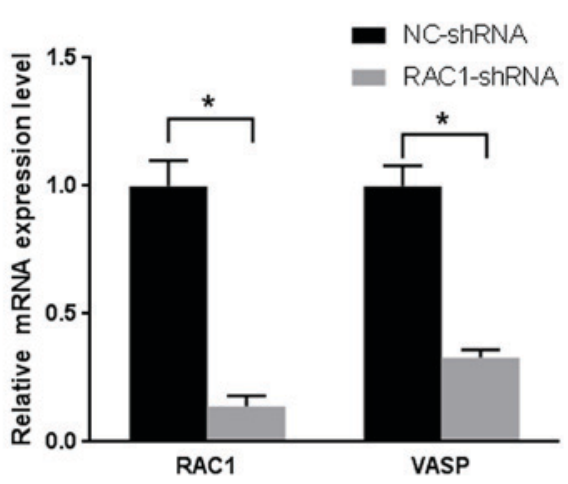

F

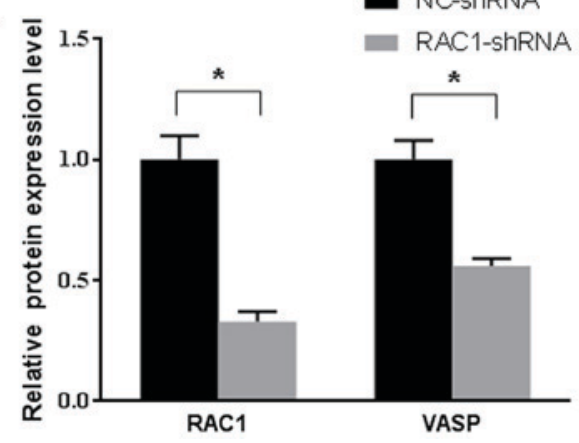

Figure 5. RAC1 and VASP expression levels following knockdown. (A) Expression level of VASP mRNA in VASP-shRNA MCF-7 cells, compared with NC-shRNA MCF-7 cells. "P<0.05. (B) Protein expression level of VASP protein in VASP-shRNA MCF-7 cells, compared with NC-shRNA MCF-7 cells. (C) Quantitative analysis of expression levels of VASP protein in panel (B). "P<0.05 compared with the control group. (D) Expression levels of VASP and RAC1 mRNA in RAC1-shRNA MCF-7 cells, compared with NC-shRNA MCF-7 cells. "P<0.05. (E) Protein expression level of VASP and RAC1 protein in RAC1-shRNA MCF-7 cells, compared with NC-shRNA MCF-7 cells. (F) Quantitative analysis of expression levels of VASP and RAC1 protein in panel (E). ${ }^{*} \mathrm{P}<0.05$ compared with the control group. RAC1, Ras-related C3 botulinum toxin substrate 1; VASP, vasodilator-stimulated phosphoprotein; NC, negative control; shRNA, short hairpin RNA. 

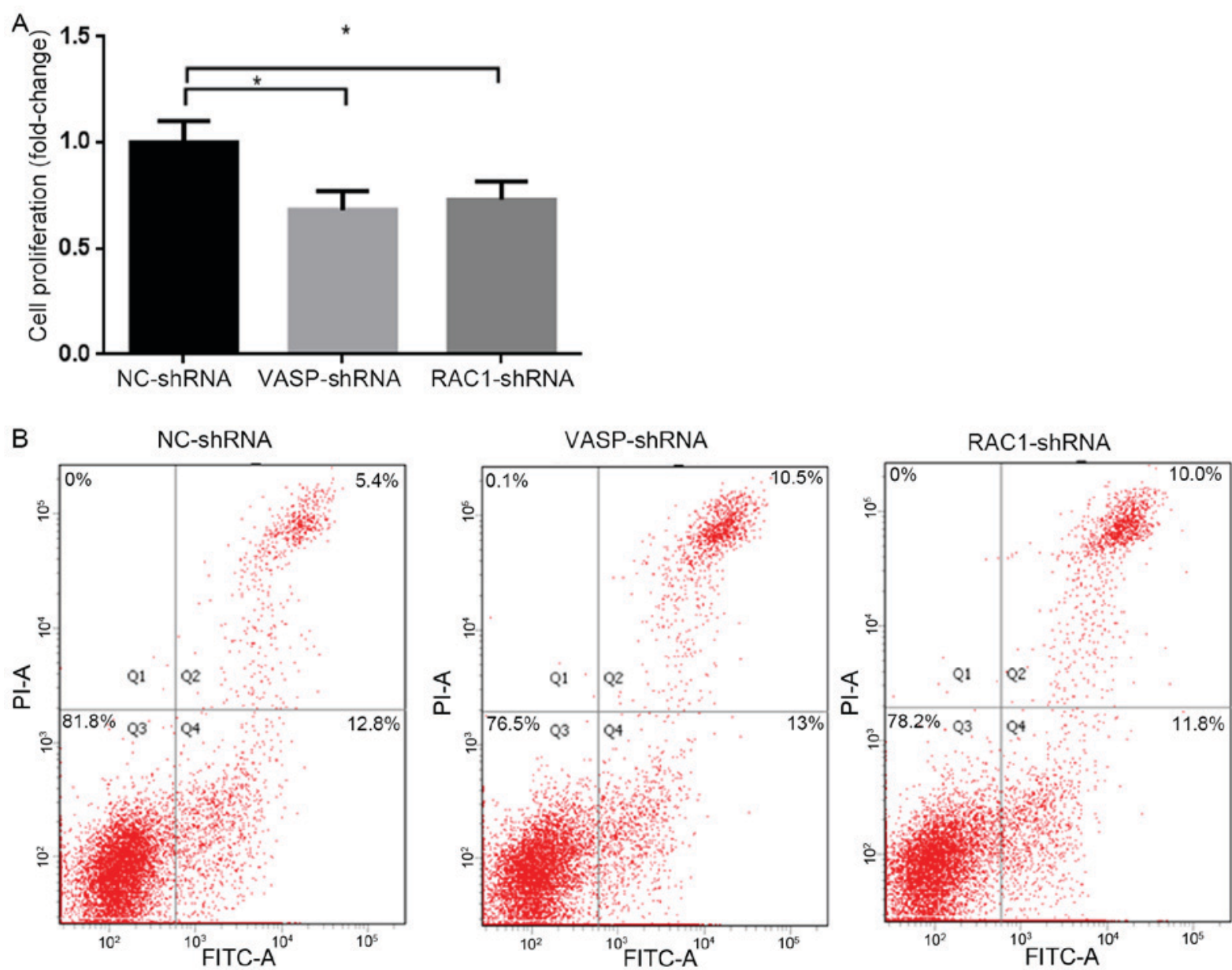

Figure 6. VASP- and RAC1-knockdown inhibits proliferation and promotes apoptosis of MCF-7 cells. (A) The fold change of VASP- and RAC1-knockdown inhibits MCF-7 cell proliferation compared with NC-shRNA MCF-7 cells. "P<0.05. (B) Interference of VASP and RAC1 promoted late apoptosis of VASP-shRNA, RAC1-shRNA MCF-7 cells, compared with the NC-shRNA MCF-7 cells * $\mathrm{P}<0.05$. RAC1, Ras-related C3 botulinum toxin substrate 1; VASP, vasodilator-stimulated phosphoprotein; NC, negative control; shRNA, short hairpin RNA; FITC, fluorescein isothiocyanate.

MCF-7 cells in the RAC1-shRNA and VASP-shRNA groups was significantly decreased compared with the NC-shRNA group, by 27 and $32 \%$, respectively $(\mathrm{P}<0.05$; Fig. $6 \mathrm{~A})$. Compared with the NC-shRNA group, the apoptotic rates of MCF-7 cells in the RAC1-shRNA and VASP-shRNA groups were increased significantly, by 4.6 and $5.1 \%$, respectively $(\mathrm{P}<0.05$; Fig. 6B).

Effect of RACl and VASP-shRNA interference on MCF-7 cell migration. In order to study the effect of downregulation of RACl and VASP expression on the migratory ability of MCF-7 cells, stable RAC1-shRNA, VASP-shRNA and NC-shRNA MCF-7 cells were cultured for $48 \mathrm{~h}$, and the 2D and 3D migration ability of the cells was analyzed using wound healing and Transwell assays. As presented in Fig. 7A, compared with MCF-7 cells in the NC-shRNA group, the 2D migratory ability of the cells in the RAC1-shRNA and VASP-shRNA groups was decreased, by 35 and $50 \%$, respectively $(\mathrm{P}<0.05$; Fig. 7B). Furthermore, the 3D migratory ability of the cells in the RAC1-shRNA and VASP-shRNA groups was decreased (Fig. 8A, by $68.9 \%(\mathrm{P}<0.05)$ and $71.1 \%$, respectively $(\mathrm{P}<0.05$; Fig. 8B). These data indicate that downregulation of RACl and VASP effectively inhibited the 2D and 3D migratory ability of MCF-7 cells.

\section{Discussion}

Rho GTPases are important downstream signaling molecules for a number of membrane surface receptors, including $\mathrm{G}$ protein-coupled, tyrosine kinase, cytokine and adhesion molecule receptors $(19,20)$. Rho GTPases act as molecular switches that regulate numerous features of cell behavior, including cell polarity, cytokinesis, particle movement, membrane trafficking, membrane transport and translocation, cell growth and transformation, and cell adhesion and motility $(21,22)$. Members of the Rho family affect cell morphology by controlling the formation of actin-dependent structures (23). RhoA is involved in the assembly of tonofibrils, $\mathrm{RAC} 1$ regulates the formation of lamellipodia and membrane wrinkling, and $\mathrm{Cdc} 42$ promotes the formation of filopodia in vascular endothelial cells (23). Furthermore, it has been demonstrated that Rho GTPases regulate carcinogenesis and tumor development (24). The mRNA and protein expression levels of RhoE have been demonstrated to be markedly increased in lung cancer tissues compared with adjacent non-tumor lung tissues, and the overexpression of RhoE in non-small cell lung cancer has been associated with smoking (25). This suggests that RhoE may serve as a molecular marker to identify high-risk individuals for lung carcinogenesis. Increased RAC1 


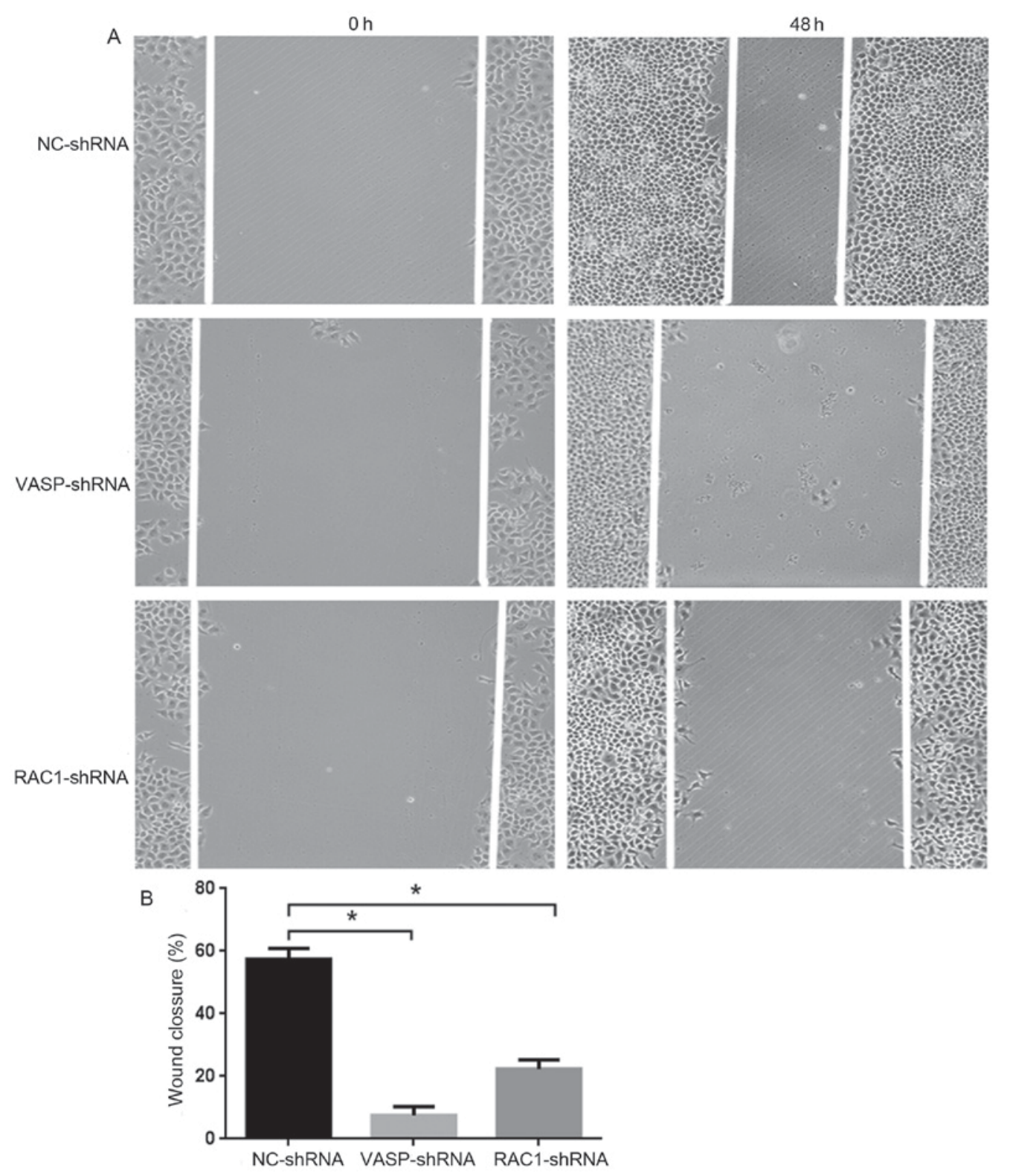

Figure 7. VASP- and RAC1-knockdown inhibits MCF-7 cell 2D migration. (A) VASP- and RAC1-knockdown inhibits MCF-7 cell migration compared with NC-shRNA MCF-7 cells. (B) Quantitative analysis of migration inhibition rate in graph (A), ${ }^{*} \mathrm{P}<0.05$ compared with the control group. RAC1, Ras-related C3 botulinum toxin substrate 1; VASP, vasodilator-stimulated phosphoprotein; NC, negative control; sh, short hairpin.

activity has been observed, in addition to the overexpression of PAK1, in urothelial carcinomas of the upper urinary tract, tumor tissue and metastatic lymph node tissue (26). This was associated with poor differentiation, local infiltration and lymph node metastasis. In view of this, the purpose of the present study was to further examine the association between $\mathrm{RACl}$ and the development of breast cancer.

Downstream effectors of activated RAC1 include a variety of actin-associated proteins, which enable actin filament polymerization at the edge of the cell lamellipodia (27). The formation of the microfilament network includes nucleation, the extension of the filament at the positive end, and branching and dissociation at the negative end. RAC1 interacts with insulin receptor substrate $\mathrm{p} 53$ and the wave protein to activate the actin-related protein $2 / 3$ complex to induce actin branch formation (28). In addition, RAC1 activates PAK1 to allow it to form a complex with, and phosphorylate, LIM domain kinase (29). LIM-kinase catalyzes phosphorylation of an $\mathrm{N}$-terminal serine residue of cofilin, thereby inactivating its F-actin-depolymerizing activity, and leading to the accumulation of actin filaments and aggregates. These results suggest that $\mathrm{RAC} 1 / \mathrm{Cdc} 42$ promotes the formation of pseudopodia and promotes cell migration by promoting actin filament polymerization and inhibition of actin filament depolymerization (30). However, as an actin-associated protein, the role of VASP in regulating the cytoskeleton is to promote the extension of actin filaments at the positive end, which is a crucial step for the formation of actin networks. Notably, there exists evidence for the presence of Ena/VASP family members (Mena, VASP and Ena-VASP-like) in the development of solid cancer (31). The intensity of Mena expression increases from premalignant to malignant lesions in various organs, including the large bowel, stomach, cervix and salivary glands $(8,32)$. Aggressive and migratory Mg-63 osteosarcoma cells contain comparatively 
A

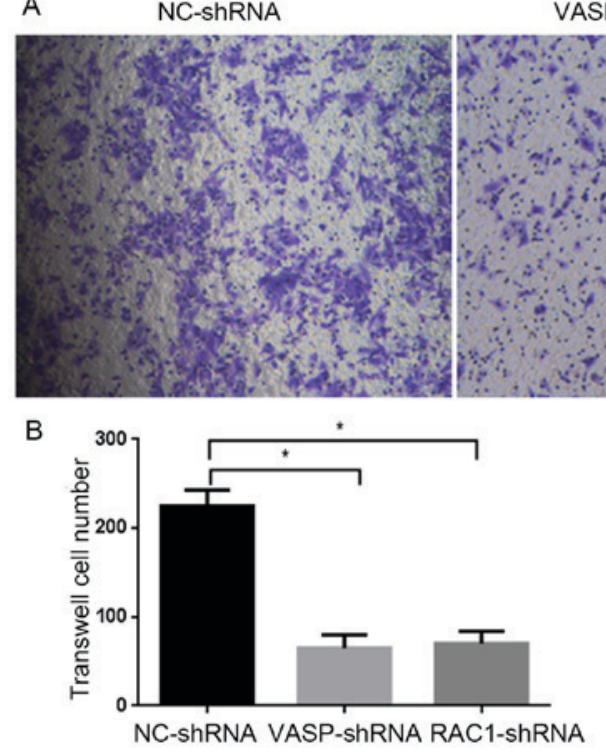

VASP-ShRNA

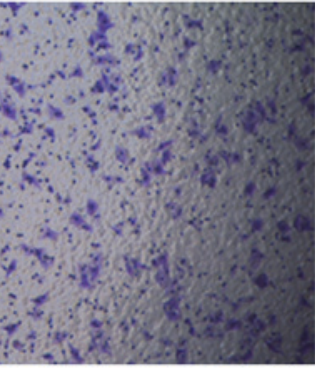

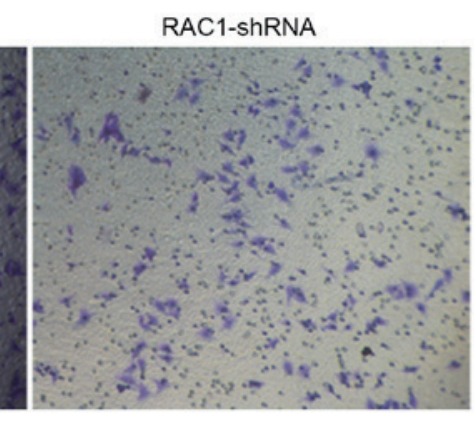

RAC1-ShRNA

.

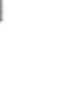

Figure 8. VASP- and RAC1-knockdown inhibits MCF-7 cell 3D migration. (A) VASP- and RAC1-knockdown inhibits the migration of VASP-shRNA and RAC1-shRNA MCF-7 cells, compared with NC-shRNA MCF-7 cells. Using an inverted phase-contrast microscope (magnification, x200; Olympus Corporation, Tokyo, Japan). (B) Quantitative analysis of migration inhibition rate in graph (A). * $\mathrm{P}<0.05$ compared with the control group. RAC1, Ras-related C3 botulinum toxin substrate 1; VASP, vasodilator-stimulated phosphoprotein; NC, negative control; shRNA, short hairpin RNA.

higher expression levels of VASP at the transcriptional and translational levels, compared with the less aggressive Saos-2 osteosarcoma cells (33). Overexpression of VASP in wild-type NIH 3 T3 cells results in metaplasia to cancer cells (34), and the increased expression level of VASP in lung adenocarcinoma correlates with lower cellular differentiation and an increased pathological stage of the tumor (8). Therefore, we hypothesized that RAC1/VASP participates in the development of breast cancer, and research was initiated to provide clinical evidence.

The present study used information from multiple databases to comprehensively analyze the association between RAC1 and VASP mRNA expression levels in breast cancer tissues and the pathological features of the patients. According to data from Oncomine, the mRNA expression levels of RAC1 and VASP in invasive ductal carcinoma tissues were significantly increased compared with control tissues. Furthermore, according to the Kaplan-Meier data, the mortality rate of patients with breast cancer at 50 months in the RAC1 higher expression level group (49\%) was increased compared with the lower expression group $(40.2 \%)$. The mortality rate of patients with breast cancer at 50 months in the high VASP expression level group was significantly increased compared with the low expression group. Furthermore, according to the data from TCGA, mRNA expression levels of RAC1 and VASP in the Normal group were the lowest, and increased gradually in the Luminal A, Luminal B, BLBC and Her-2 enrichment subtype groups. The mRNA expression levels of RAC1 and VASP gradually increased in breast cancer tissues of stages I, II, III and IV. These data indicate that the high expression levels of RAC1 and VASP in breast cancer are associated with low cellular differentiation, high pathological stage and more aggressive tumor subtypes. High VASP mRNA expression levels were positively associated with a poor overall survival time in patients with breast cancer. However, the mechanism of RAC1/VASP function in the progression of breast cancer development requires further investigation and remains to be fully elucidated.

For further examination of the role of RAC1 and VASP in the proliferation and migration of breast cancer cells, RAC1 or VASP-knockdown cell lines established. In the RAC1-shRNA or VASP-shRNA MCF-7 cells, the protein expression levels of RAC1 and VASP were downregulated, the proliferation rates were decreased and the migratory abilities decreased. These data indicated that RAC1 and VASP promoted breast cancer progression and development. Notably, it was observed that the protein expression level of VASP was decreased in RAC1-shRNA MCF-7 cells. However, the mechanism through which $\mathrm{RAC} 1$ regulates VASP remains unclear. PAK is a RAC1 downstream target molecule, and when the its serine 21 site is phosphorylated by $\mathrm{PKG}, \mathrm{PAK} / \mathrm{NCK}$ binding is inhibited and PAK/VASP binding is enhanced, in order to promote human umbilical vein endothelial cell motility (30). The binding takes place between the proline-rich region of PAK and the EVH1 domain of VASP $(3,35)$. These data may act as a foundation on which further investigations may be based.

In conclusion, RAC1 and VASP may act as proto-oncogenes in the development of breast cancer, and VASP may act as a downstream target of RAC1 to promote the proliferation and migration of breast cancer cells.

\section{Acknowledgements}

Not applicable.

\section{Funding}

National Natural Science Foundation of China (grant no. 81572943). 


\section{Availability of data and materials}

The datasets used and/or analyzed during the current study are available from the corresponding author on reasonable request.

\section{Authors' contributions}

YT, LX and LW designed the study and wrote the manuscript, $\mathrm{XX}, \mathrm{KL}$ and $\mathrm{YM}$ analyzed the data, and $\mathrm{YG}, \mathrm{DW}$ and $\mathrm{YH}$ performed the cell experiments.

\section{Ethics approval and consent to participate}

Not applicable.

\section{Consent for publication}

Not applicable.

\section{Competing interests}

The authors declare that they have no competing interests.

\section{References}

1. Chen W, Zheng R, Baade PD, Zhang S, Zeng H, Bray F, Jemal A Yu XQ and He J: Cancer statistics in China, 2015. CA Cancer J Clin 66: 115-132, 2016.

2. Lopez-Marure R, Contreras PG and Dillon JS: Effects of dehydroepiandrosterone on proliferation, migration, and death of breast cancer cells. Eur J Pharmacol 660: 268-274, 2011.

3. Krause M, Dent EW, Bear JE, Loureiro JJ and Gertler FB Ena/VASP proteins: Regulators of the actin cytoskeleton and cell migration. Annu Rev Cell Dev Biol 19: 541-564, 2003.

4. Ali M, Rogers LK and Pitari GM: Serine phosphorylation of vasodilator-stimulated phosphoprotein (VASP) regulates colon cancer cell survival and apoptosis. Life Sci 123: 1-8, 2015.

5. Pula G and Krause M: Role of Ena/VASP proteins in homeostasis and disease. Handb Exp Pharmacol: 39-65, 2008.

6. Sechi AS and Wehland J: ENA/VASP proteins: Multifunctional regulators of actin cytoskeleton dynamics. Front Biosci 9 : 1294-1310, 2004.

7. Jayakumar T, Lin KC, Lu WJ, Lin CY, Pitchairaj G, Li JY and Sheu JR: Nobiletin, a citrus flavonoid, activates vasodilator-stimulated phosphoprotein in human platelets through non-cyclic nucleotide-related mechanisms. Int J Mol Med 39: 174-182, 2017.

8. Dertsiz L, Ozbilim G, Kayisli Y, Gokhan GA, Demircan A and Kayisli UA: Differential expression of VASP in normal lung tissue and lung adenocarcinomas. Thorax 60: 576-581, 2005.

9. Cardama GA, Gonzalez N, Maggio J, Menna PL and Gomez DE: Rho GTPases as therapeutic targets in cancer (Review). Int J Oncol 51: 1025-1034, 2017.

10. Orgaz JL, Herraiz C and Sanz-Moreno V: Rho GTPases modulate malignant transformation of tumor cells. Small GTPases 5: e29019,2014.

11. Kazanietz MG and Caloca MJ: The Rac GTPase in cancer: From old concepts to new paradigms. Cancer Res 77: 5445-5451, 2017.

12. Zuzga DS, Pelta-Heller J, Li P, Bombonati A, Waldman SA and Pitari GM: Phosphorylation of vasodilator-stimulated phosphoprotein Ser239 suppresses filopodia and invadopodia in colon cancer. Int J Cancer 130: 2539-2548, 2012.

13. Espina C,Céspedes MV,García-Cabezas MA,Gómez del PulgarMT, Boluda A, Oroz LG, Benitah SA, Cejas P, Nistal M, Mangues R and Lacal JC: A critical role for Racl in tumor progression of human colorectal adenocarcinoma cells. Am J Pathol 172: 156-166, 2008.

14. Fryer BH, Wang C, Vedantam S, Zhou GL, Jin S, Fletcher L, Simon MC and Field J: cGMP-dependent protein kinase phosphorylates p21-activated kinase (Pak) 1, inhibiting Pak/Nck binding and stimulating Pak/vasodilator-stimulated phosphoprotein association. J Biol Chem 281: 11487-11495, 2006.
15. Györffy B, Lanczky A, Eklund AC, Denkert C, Budczies J, Li Q and Szallasi Z: An online survival analysis tool to rapidly assess the effect of 22,277 genes on breast cancer prognosis using microarray data of 1,809 patients. Breast Cancer Res Treat 123: 725-731, 2010.

16. Parker JS, Mullins M, Cheang MC, Leung S, Voduc D, Vickery T, Davies S, Fauron C, He X, Hu Z, et al: Supervised risk predictor of breast cancer based on intrinsic subtypes. J Clin Oncol 27: 1160-1167, 2009.

17. Ye J, Wang W, Xu L, Duan X, Cheng Y, Xin L, Zhang H, Zhang S, Li T and Liu Y: A retrospective prognostic evaluation analysis using the 8th edition of American Joint Committee on Cancer (AJCC) cancer staging system for luminal A breast cancer. Chin J Cancer Res 29: 351-360, 2017.

18. Penault-Llorca F: Comments on the new American Joint Committee on Cancer TNM staging for breast cancer. What's new for the pathologist? Ann Pathol 23: 492-495, 2003 (In French).

19. Aznar S and Lacal JC: Rho signals to cell growth and apoptosis. Cancer Lett 165: 1-10, 2001.

20. Bar-Sagi D and Hall A: Ras and Rho GTPases: A family reunion. Cell 103: 227-238, 2000.

21. Schaefer A, Reinhard NR and Hordijk PL: Toward understanding RhoGTPase specificity: Structure, function and local activation. Small GTPases 5: 6, 2014.

22. Zandvakili I, Lin Y, Morris JC and Zheng Y: Rho GTPases: Antior pro-neoplastic targets? Oncogene 36: 3213-3222, 2017.

23. Hall A: Rho GTPases and the actin cytoskeleton. Science 279: 509-514, 1998.

24. Roux P, Gauthier-Rouviere C, Doucet-Brutin S and Fort P: The small GTPases Cdc42Hs, Rac1 and RhoG delineate Raf-independent pathways that cooperate to transform NIH3T3 cells. Curr Biol 7: 629-637, 1997.

25. Cuiyan Z, Jie H, Fang Z, Kezhi Z, Junting W, Susheng S, Xiaoli F, Ning L, Xinhua M, Zhaoli C, et al: Overexpression of RhoE in non-small cell lung cancer (NSCLC) is associated with smoking and correlates with DNA copy number changes. Cancer Biol Ther 6: 335-342, 2007

26. Kamai T, Shirataki H, Nakanishi K, Furuya N, Kambara T, Abe H, Oyama T and Yoshida K: Increased Racl activity and Pak1 overexpression are associated with lymphovascular invasion and lymph node metastasis of upper urinary tract cancer. BMC Cancer 10: 164, 2010.

27. Li YL, Shao M and Shi DL: Rac1 signalling coordinates epiboly movement by differential regulation of actin cytoskeleton in zebrafish. Biochem Biophys Res Commun 490: 1059-1065, 2017.

28. Lim KB, Bu W, Goh WI, Koh E, Ong SH, Pawson T, Sudhaharan T and Ahmed S: The Cdc42 effector IRSp53 generates filopodia by coupling membrane protrusion with actin dynamics. J Biol Chem 283: 20454-20472, 2008.

29. Dawid IB, Breen JJ and Toyama R: LIM domains: Multiple roles as adapters and functional modifiers in protein interactions. Trends Genet 14: 156-162, 1998.

30. Edwards DC, Sanders LC, Bokoch GM and Gill GN: Activation of LIM-kinase by Pak1 couples Rac/Cdc42 GTPase signalling to actin cytoskeletal dynamics. Nat Cell Biol 1: 253-259, 1999.

31. Zhang YT, Xu LH, Lu Q, Liu KP, Liu PY,Ji F, Liu XM, Ouyang DY and He XH: VASP activation via the Go13/RhoA/PKA pathway mediates cucurbitacin-B-induced actin aggregation and cofilin-actin rod formation. PLoS One 9: e93547, 2014.

32. Gurzu S, Ciortea D, Ember I and Jung I: The possible role of Mena protein and its splicing-derived variants in embryogenesis, carcinogenesis, and tumor invasion: A systematic review of the literature. Biomed Res Int 2013: 365192, 2013.

33. Wu G, Wei L, Yu A, Zhang M, Qi B, Su K, Hu X and Wang J: Vasodilator-stimulated phosphoprotein regulates osteosarcoma cell migration. Oncol Rep 26: 1609-1615, 2011.

34. Liu K,LiL,Nisson PE,GruberC,Jessee J and Cohen SN:Reversible tumorigenesis induced by deficiency of vasodilator-stimulated phosphoprotein. Mol Cell Biol 19: 3696-3703, 1999.

35. Greenwood AI, Kwon J and Nicholson LK: Isomerase-catalyzed binding of interleukin-1 receptor-associated kinase 1 to the EVH1 domain of vasodilator-stimulated phosphoprotein. Biochemistry 53: 3593-3607, 2014.

This work is licensed under a Creative Commons Attribution-NonCommercial-NoDerivatives 4.0 International (CC BY-NC-ND 4.0) License. 